\title{
The Role of Environmental Law in Ecological Resources Protection under the Background of Wireless Communication and Artificial Intelligence
}

\author{
Junyan $\mathrm{Wu} \mathbb{D}^{1}$ and Wenjun Tang $\mathbb{D}^{2}$ \\ ${ }^{1}$ Law College, Chengdu University, Chengdu 610106, China \\ ${ }^{2}$ Higher Research Institute (Shenzhen) of University of Electronic Science and Technology, Shenzhen 518110, China \\ Correspondence should be addressed to Junyan Wu; wu_junyan@outlook.com
}

Received 22 November 2021; Revised 11 January 2022; Accepted 21 January 2022; Published 28 February 2022

Academic Editor: M Pallikonda Rajasekaran

Copyright (c) 2022 Junyan $\mathrm{Wu}$ and Wenjun Tang. This is an open access article distributed under the Creative Commons Attribution License, which permits unrestricted use, distribution, and reproduction in any medium, provided the original work is properly cited.

\begin{abstract}
With the rapid development of China's economy and the continuous improvement of people's living standards, people pay more and more attention to environmental problems. It faces great challenges in energy, resources, and environmental protection. In recent years, people have realized that environmental pollution is a global problem. Therefore, the protection of ecological resources is an important topic in today's society. Based on wireless communication and artificial intelligence, this paper studies the role of environmental law in the protection of ecological resources. Firstly, this paper explains and introduces the concept and purpose of environmental rule of law, then expounds the importance of ecological resource protection, studies the wireless communication and artificial intelligence technology, designs the wireless communication and framework and the structure of artificial intelligence system, and adopts the questionnaire survey method. This paper studies and investigates an ecological resource protection area in China, and tests wireless communication technology and artificial intelligence technology. Finally, the test results show that the resources in the ecological nature reserve are evenly distributed, but there are still problems of overexploitation of resources and poor management. Each module of the ecological resource protection system based on artificial intelligence operates normally, the delay time and operation time are between 1 and $3 \mathrm{~s}$, and the amount of memory is normal. In addition, the feasibility, effectiveness, and real-time performance of each module are more than $90 \%$. All these show that the system can meet the requirements of normal operation.
\end{abstract}

\section{Introduction}

With the progress of human society, people pay more and more attention to environmental problems, and ecological resources are an important part of them, which plays an important role in protecting the ecological environment $[1,2]$. However, due to the development of science and technology, the gradual enhancement of human awareness of environmental protection, the imperfection of China's legal system, the lack of relevant environmental knowledge, and insufficient publicity, many people are unable to understand the latest and real information. Under such a background, the research on wireless communication, artificial intelligence, and ecological resource protection has become a highly concerned topic in the world $[3,4]$.
Many scholars have conducted relevant research on ecological resources protection. Some scholars combined the new development theory of the Yangtze River with the successful practical experience of urban ecological embankment construction and put forward a new concept of urban ecological embankment planning and design. Then, the typical section of urban ecological embankment is introduced, and the construction of urban ecological embankment is analyzed as an example. Finally, some challenges faced by urban ecological embankment construction are analyzed, and the research results can provide guidance for the planning and design of urban ecological embankments $[5,6]$. Other scholars plan and manage the protection of nature reserves based on computer technology $[7,8]$. The above research lays a foundation for the study of 
the role of environmental law in the protection of ecological resources based on wireless communication and artificial intelligence.

This paper will study its mechanism and influencing factors from two aspects of artificial intelligence and environmental protection, combined with the status quo and deficiencies of laws and regulations in the field of environmental protection in the country, and then consider the new methods existing in the context of the development of artificial intelligence and ZigBee wireless communication technologies. Corresponding measures and suggestions to strengthen the ecological environment and resource protection.

\section{Discussion on the Protection of Ecological Resources by Environmental Law under the Background of Wireless Communication and Artificial Intelligence}

\subsection{Environmental Rule of Law}

2.1.1. Concept. Various social norms, whether moral norms, legal norms, customs, religions, and other norms, exist as ideology and are produced and developed for the needs of people's social life in social practice $[9,10]$. Solving the existing problems, stabilizing the existing order, and ensuring the healthy development of human society are the fundamental objectives of all social norms. Any major problem in human society will have a significant impact on the traditional social order, and there is an urgent need to establish a new social order to adapt to the evolution of social structure $[11,12]$.

The emergence and aggravation of environmental problems highlight the necessity of reforming and improving environmental protection laws and regulations [13-16]. On the surface, environmental problems are caused by poor management of the relationship between man and nature, but fundamentally, environmental problems include a variety of complex factors such as population, culture, system, politics, economy, and ideology. The development of the environmental crisis is the result of the combined effect of the above factors and the development of industrial civilization. The legal system, including environmental legislation, enforcement, judiciary, compliance, and legal oversight, has a static meaning. By establishing the basic theory of the rule of law and the corresponding operating mechanism, it strives to coordinate human nature in different backgrounds and processes such as legislation, law enforcement, and judiciary [17-19].

\subsubsection{Purpose of Environmental Rule of law}

(1) Environmental Law Meets the Regulatory Requirements in the Field of Environment. The main goal and pursuit of the value of environmental rule of law are to meet the requirements of the order in the field of environment. To solve environmental problems, it is far from enough to rely on moral education alone. Certain mandatory measures must be taken to ensure effective implementation. The fundamental measure of the legalization of environmental law is to promote moral norms into legal norms through legislation. The rule of law interferes and restricts the relationship between man and nature. The implementation of law controls people's ideology and conscience in using nature and obtaining resources. It is in this way that legal power is used to regulate, adapt, and repair the relationship between man and nature, repair the seriously damaged relationship between man and nature, control environmental problems, and improve the current situation of environmental policies.

(2) Legalization of Environmental Law to Create a Social Development Model of Harmonious Coexistence between Man and Environment. The fundamental purpose of environmental law is to create a social development model in which people live in harmony with the environment. The environmental rule of law uses a variety of legal means to intervene in human actions and omissions in the process of interaction with the natural environment, so as to standardize human behavior, so as to solve the environmental crisis and create the environment. Its fundamental purpose and direction are to summarize the successful experience of human beings in abiding by the laws of nature in long-term life practice and the lessons of punishment for violating the laws of nature and bring them into the process of environmental construction.

(3) Environmental Rule of Law is Helpful to Understand the Spiritual Value of Environment to Human Beings. The environmental rule of law helps people to carry out their material activities scientifically while realizing the spiritual value of the environment to mankind. The rule of law in environmental law embodies the rules and requirements of environmental ethics in all links and processes of the construction of the rule of law and transforms mild morality into law supported by coercion. In people's hearts, it forces people to develop production scientifically and reasonably and improve the quality of life, without ignoring the care and protection of the natural environment. In a good and orderly living and production environment in which people live in harmony with nature, people can have a steady stream of resources provided by nature, and at the same time experience the rich and diverse spirit of nature. People pursue the greatest material satisfaction, but also the greatest spiritual satisfaction.

\subsection{Protection of Ecological Resources}

2.2.1. Current Ecological Environment Problems. The importance of ecological and environmental issues lies in the negative feedback of people. Humans influence the ecological environment through sustainable production and living. Ecological environmental problems include native environmental problems and man-made environmental problems. The current environmental and ecological dilemmas facing my country are mainly due to the pollution 
of life and production, and the serious damage to the ecological environment, including regional haze, soil erosion, land destruction, and grassland degradation. In recent years, the continuous attack of severe and abnormal weather phenomena and the aggravation of ecological damage and pollution also reminds us that we must soberly realize that our country is still a country that accelerates the improvement of the ecological environment as a whole.

2.2.2. Significance of Environmental Development to Ecological Resource Protection. Ecological resources mean scarcity and irreversibility. The destruction of natural ecology will cause serious consequences and cannot be restored. As wealth is left by nature to mankind, natural ecological resources must be fully protected. These must also be checked when developing and utilizing ecological resources and can be constructed only after reasonable planning. With the acceleration of urbanization and the destruction of population, a large area of ecological resources has been destroyed. The importance of environmental law is to better protect natural ecological resources, avoid major damage, and carry out appropriate protection planning to avoid future losses. Due to people's weak awareness of protection, haze in the air, river depletion, and serious pollution, deforestation leads to bare vegetation and soil erosion on the mountain. Environmental law can better protect the natural ecological environment. Leave the beautiful ecological environment to the next generation for future generations to enjoy.

\subsection{Wireless Communication and Artificial Intelligence in the Context of Big Data}

\subsubsection{Artificial Intelligence}

(1) Concept. Artificial intelligence is the result of technological development and the embodiment of human wisdom. Artificial intelligence can simulate human behavior and thoughts through wireless communication technology, which is the result of high-tech technology theory and application. Artificial intelligence can replace humans in some simple, dangerous jobs. Artificial intelligence mainly includes information representation and thinking simulation, data processing, machine learning and information acquisition, computer vision, and natural language understanding.

(2) Algorithm. The algorithm idea of K-means algorithm is roughly explained as follows: first randomly select $\mathrm{K}$ points from many samples as "cluster centers," and calculate the distance between each sample and the K "cluster centers" one by one. If a sample is closest to a "cluster center" point, the sample is divided into the cluster where the "cluster center" is located. Key points of the K-means algorithm:

Minkowski distance is

$$
\operatorname{dist}_{m k}\left(\lambda^{\alpha}, \lambda^{\beta}\right)=\left(\sum_{\phi=1}^{n}\left|\lambda_{\phi}^{\alpha}-\lambda_{\phi}^{\beta}\right|\right)^{1 / \mu} .
$$

Euclidean distance:

$$
\operatorname{dist}_{\text {ed }}\left(\lambda^{\alpha}, \lambda^{\beta}\right)=\left\|\lambda^{\alpha}, \lambda^{\beta}\right\| .
$$

Manhattan distance:

$$
\operatorname{dist}_{\operatorname{man}}\left(\lambda^{\alpha}, \lambda^{\beta}\right)=\left\|\lambda^{\alpha}-\lambda^{\beta}\right\| .
$$

2.3.2. Wireless Communication Technology. At the beginning of the 21st century, many electronic companies established the Zig Bee alliance to develop new wireless communication technologies. The name Zig Bee comes from the dance of bees. After the release of Zig Bee, it has attracted the attention of the market. Many companies have joined the Zig Bee alliance. At present, more than 100 enterprises have joined the alliance and launched their own products, which has played a positive role in promoting the development of this technology. ZigBee is a communication protocol developed by the ZigBee Alliance, which is based on IEEE 802.15.4 protocol. IEEE 802 protocol defines the physical layer and MAC layer, and ZigBee defines the network layer and application layer. Zig Bee mainly works in the $2.4 \mathrm{GHz}$ band and has the characteristics of low speed, low power consumption, low cost, short delivery time, flexible network topology, and so on. The network protocol architecture of ZigBee consists of a physical layer (PHY), media access layer (MAC), network layer (NWK), and application layer (APL). The specific structure is shown in Figure 1.

\section{Experiment}

3.1. Artificial Intelligence System for Ecological Environment and Resource Protection. Figure 2 shows the ecological resource protection system developed by the combination of wireless communication technology and artificial intelligence. The main task of system function design is to divide the system into several main functional modules according to the actual needs, determine the relationship between each module, and describe each module. The system and operation-related hardware, software, and environment suitable for system development and operation are configured according to the system design objectives. Specific documents, manuals, and data required for each stage of system development must be attached with specific instructions and regulations. In addition, it also includes the quality of the system itself, the performance of the system, and the security of system estimation and regulation.

\subsection{Database Design Principles}

3.2.1. Standardization Principle. Due to the numerous data sources of ecological resources and the complexity of the database, it is necessary to solve the problem that the 


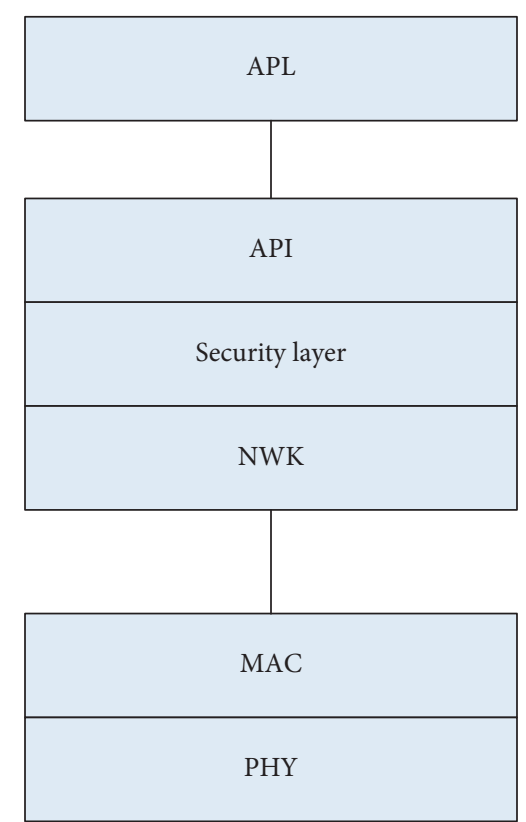

FIgURE 1: ZigBee wireless communication framework diagram.

establishment of the database corresponds to the specifications and technical specifications of relevant departments and units, for example, data classification, accuracy requirements, and data symbols.

3.2.2. Principle of Reasonable Structure. In order to ensure data integrity, security, and consistency, database design must adhere to the principle of rational structure. A reasonable data structure is conducive to data maintenance and can reduce data redundancy.

3.2.3. Layered Design Principle. In order to make the database structure clear and hierarchical, the database adopts a hierarchical design to manage data, which is convenient for personnel to design, develop, manage, and maintain the database. The hierarchical database structure has little impact on the application of data when changing the storage structure and storage mode. The storage structure has the characteristics of maintenance and operation.

3.2.4. Multiscale Coordination Principle. When spatial data is stored in a multiscale database, duplicate data collection should be avoided as far as possible. When establishing a multiscale spatial database, we must ensure the logical connection between database systems, and finally, establish a logically transparent multiscale database.

3.3. Experimental Process. This paper will use the method of a questionnaire survey to select the country's ecological resource reserves as the test object. The experimental area includes water resources, forests, and other types of environmental ecological resources, which are suitable for environmental status monitoring. Investigate and study the problems faced by resource protection in this area and use wireless communication technologies such as ZigBee gateways and sensor nodes to cooperate and monitor the area is being adjusted. Evaluate the performance of the ecological resource protection system based on artificial intelligence, and then conduct a comprehensive analysis of these problems to further improve the system.

\section{Discussion}

4.1. Distribution of Natural Resources in Ecological Resource Reserve. According to the survey, the whole mountain in the nature reserve is composed of granite, and all surface water can be seen and utilized. Table 1 shows that many streams and springs in the nature reserve converge into rivers. There are many waterfalls along the river in the nature reserve, including more than 20 waterfalls above 20 meters, more than 10 waterfalls between 60 and 100 meters, and 4 and 5 waterfalls above 100 meters. On average, there are four floods in mountainous areas every year, mainly from June to August. Floods usually last for three hours after the rain stops. There are mainly six types of soil, namely brown soil, which is mainly distributed in areas above 800 meters. Yellowish-brown clay is mainly distributed in the hills on both banks of the river and the transition zone from flat mountain to plain. Coarse bone soil is mainly distributed in mountainous and hilly rock outcrops. Solid bone soil is mainly found in bare mountainous areas in flat hilly areas. Tidal soil is mainly distributed in the alluvial plain and river plain on both banks of the river. In the flora of this nature reserve, most areas are composed of conifers, deciduous trees, shrubs, grasses, ferns, and mosses. The nature reserve is located in the strategic position of the mountains, with complex terrain and at the intersection of low mountains and plains. Rich in animal and plant resources, there are nearly 1000 species of plants. All this ensures the growth and reproduction of plants and animals.

4.2. Wireless Communication Test. The steps of the wireless communication test are as follows: first, perform multiple tests at each set distance point. If the ZigBee gateway node can receive information, it can be determined that the detection area can communicate. There are two types of transmission methods, namely, direct transmission and indirect transmission. If the sensor sends information directly to the ZigBee gateway node, this transmission method is called direct transmission. If the sensor ignores a ZigBee gateway node and sends the information to the next ZigBee gateway node, this transmission method is called indirect transmission. The test results are shown in Table 2.

Through the communication distance test shown in Figure 3, we can see that in the case of any obstacle, the effective wireless communication distance of the sensor node after jumping can reach about $241 \mathrm{~m}$, which is the requirement of system application. 


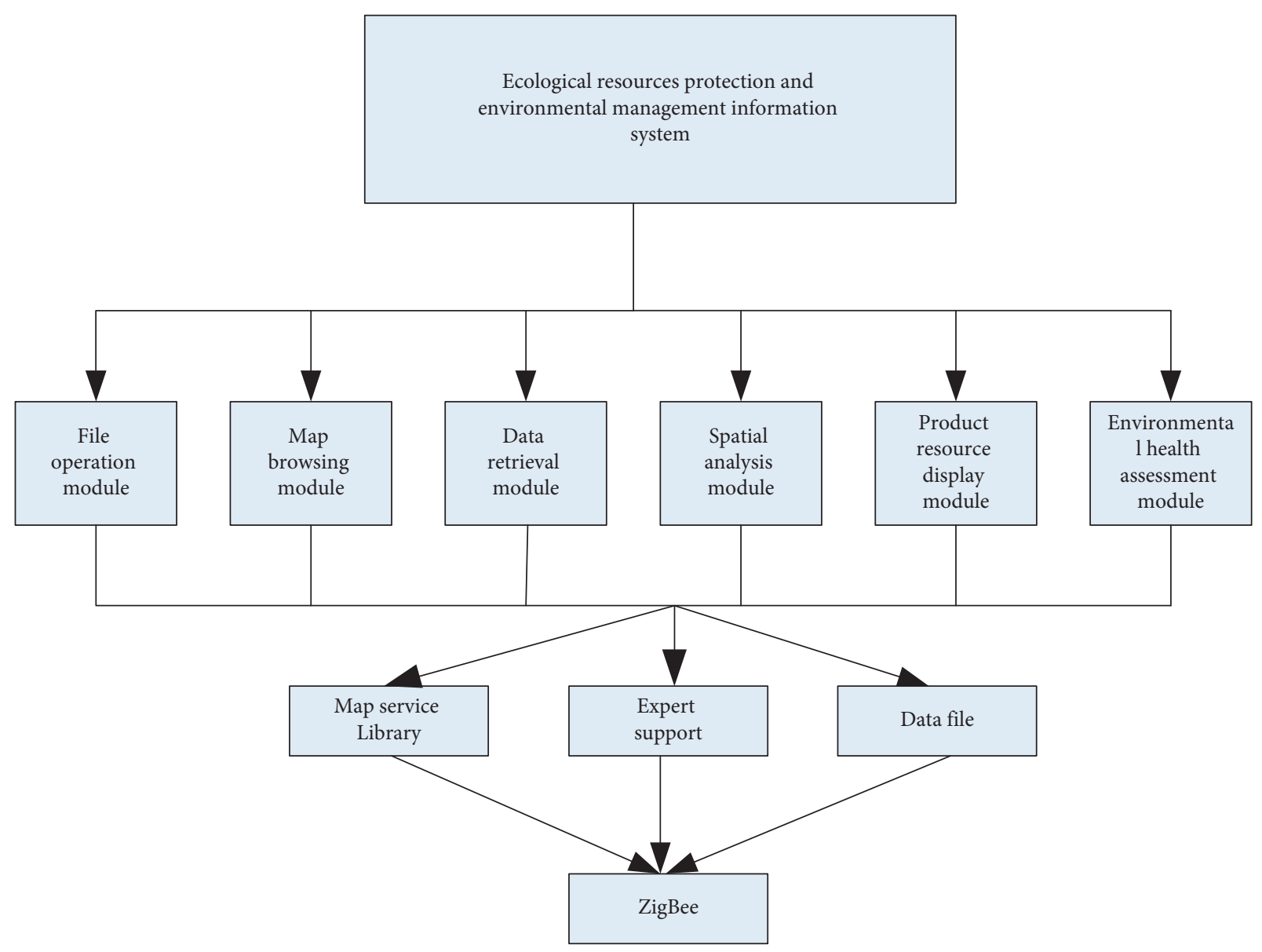

Figure 2: Ecological resource protection system.

TABLE 1: Distribution of ecological and natural resources.

\begin{tabular}{lcccc}
\hline & \multirow{2}{*}{ Water resource (\%) } & Soil resource (\%) & Plant resources (\%) & Animal resources (\%) \\
\hline Distribution & 46 & 81 & 67 & 58 \\
\hline
\end{tabular}

TABle 2: Communication distance test.

\begin{tabular}{lccc}
\hline Number of times & Measuring distance $(\mathrm{m})$ & \multicolumn{2}{c}{ Whether a signal is received } \\
There are obstacles \\
\hline 1 & 212 & No obstacles & YES \\
2 & 251 & YES & NO \\
3 & 217 & YES & YES \\
4 & 243 & YES & NO \\
5 & 238 & YES & YES \\
\hline
\end{tabular}

4.3. Performance and Practicability Test of Artificial Intelligence System. This paper also tests the performance of the ecological resource protection system based on artificial intelligence and observes the feasibility, effectiveness, and real-time performance of each module of the system. It can be seen from Tables 3 and 4 and Figure 4 that each module of the system operates normally, the delay time and operation time are between 1 and $3 \mathrm{~s}$, and the memory occupation is normal. In addition, the feasibility, effectiveness, and real-time performance of each module are more than $90 \%$. All these show that the system can meet the requirements of normal operation. 


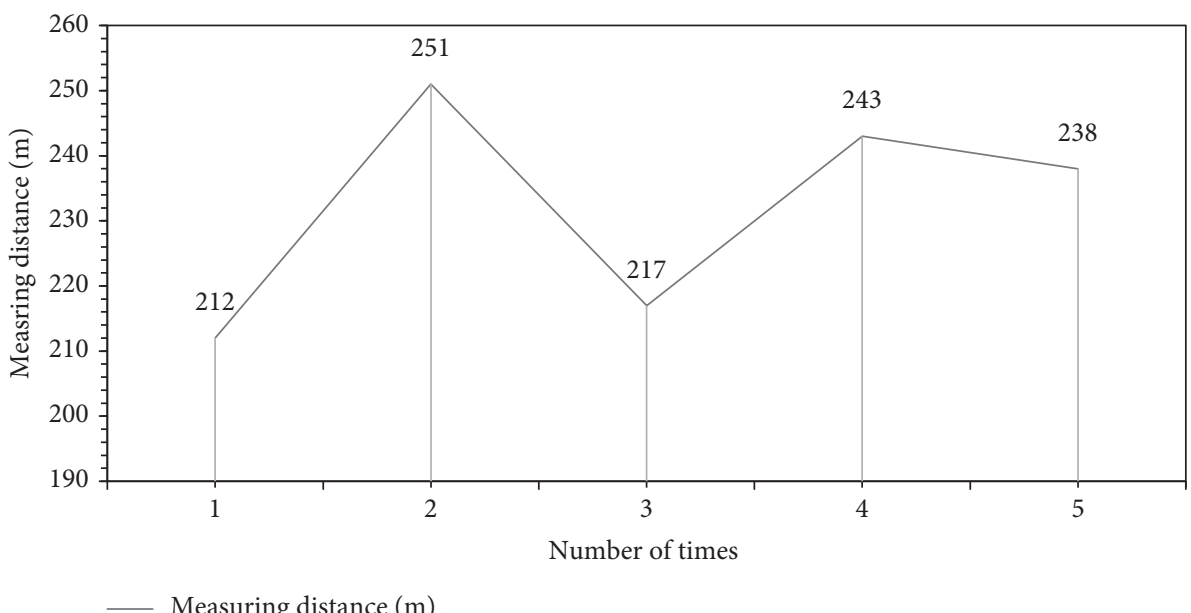

Figure 3: Measuring distance.

TABLE 3: System performance detection data.

\begin{tabular}{lccc}
\hline Number of monitoring times & Delay time $(\mathrm{s})$ & Operate time $(\mathrm{s})$ & Accounting for the amount of memory $(\mathrm{k})$ \\
\hline 1 & 1 & 2 & 3153 \\
2 & 2 & 3 & 3252 \\
3 & 1 & 2 & 3103 \\
4 & 3 & 4 & 3501 \\
5 & 2 & 3 & 3424 \\
\hline
\end{tabular}

TABle 4: Practical detection data.

\begin{tabular}{lccc}
\hline Module & Feasibility (\%) & Availability (\%) & Real-time (\%) \\
\hline File operation module & 88 & 92 & 91 \\
Map browsing module & 90 & 96 & 100 \\
Data retrieval module & 94 & 97 & 100 \\
Spatial analysis module & 92 & 94 & 100 \\
Product resource display module & 92 & 97 & 100 \\
Environmental health assessment module & 93 & & 100 \\
\hline
\end{tabular}

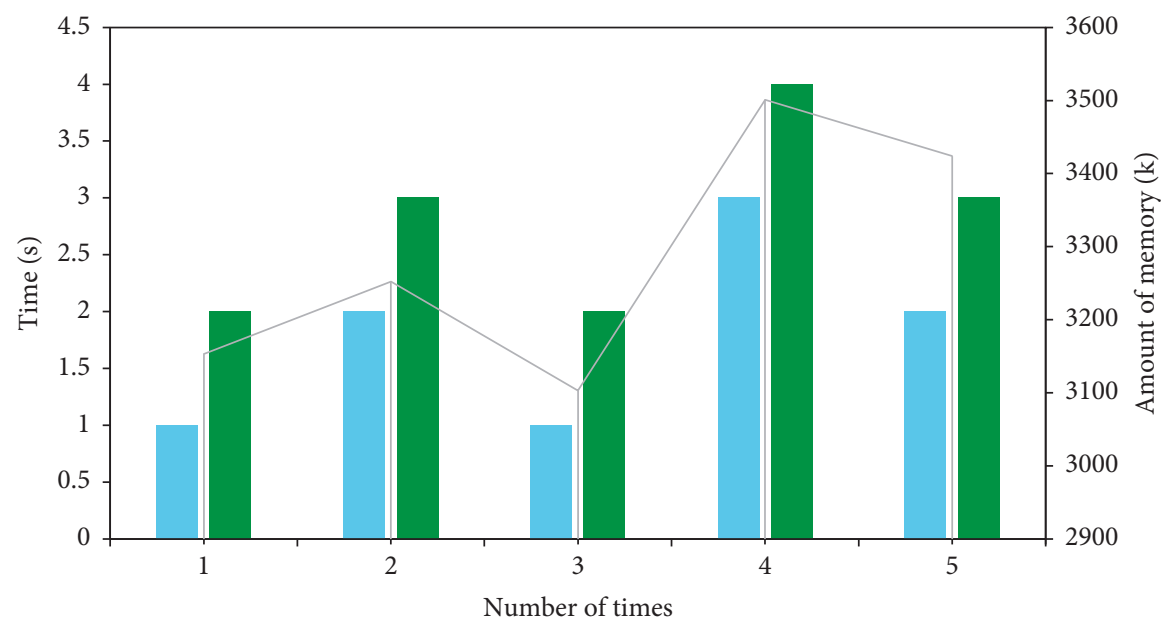

Delay time (s)

Operate time (s)

_ Accounting for the amount of memory (k)

FIgURe 4: Performance detection. 


\section{Conclusion}

With the rapid development of science and technology, environmental problems around the world are becoming more and more serious, and human beings are facing huge challenges. In this case, the research of wireless communication and artificial intelligence is of great significance to the protection of ecological resources. This paper adopts the method of questionnaire survey and equipment collection, selects the country's ecological resource protection area as the test object, uses ZigBee gateway, sensor node, and other technologies to conduct sample research, combines artificial intelligence technology, and takes a certain area as an example to analyze the use of intelligent robots in mobile terminals. The resulting series of new technologies are applied on the impact and harm of the ecological environment and ecosystems, how to better play their role is explored, and the theoretical model data collected through questionnaires are verified. The conclusion is that the control of environmental pollution needs to rely on scientific and technological innovation. Only perfect laws and regulations can effectively solve ecological and social problems.

\section{Data Availability}

The datasets used and/or analyzed during the current study are available from the corresponding author on reasonable request.

\section{Conflicts of Interest}

The authors declare that there are no conflicts of interest.

\section{Acknowledgments}

Research on Construction and Implementation Guarantee of Plaintiff System of Civil Environment-related Litigation Under the Synergy Theory, Social Science Study and Planning Program of Sichuan (Program No: SC20B001); Research on Collaborative Legislation of Jointly Building a West Financial Center in Chendu-Chongqing Region, Social Science Study and Planning Program of Sichuan (Program No: SC21ZDCY008); and Reflections and Reconstruction of the Legal System of Medical Informed Consent in China: Legislative Investigation, Troubleshooting and Development Path, The Medical and Legal Health Research Center of Sichuan (Program No: YF21-Y13).

\section{References}

[1] S. Du and X. Y. Dong, "Study on the protection and development of traditional villages under the background of constructing well off villages with ecological civilization in Gannan Tibetan areas," Journal of Lanzhou Jiaotong University, vol. 38, no. 1, pp. 19-25, 2019.

[2] C. Zhao, S. M. Zhong, and X. J. Zhang, "Novel results on nonfragile sampled-data exponential synchronization for delayed complex dynamical networks," International Journal of Robust and Nonlinear Control, vol. 30, no. 9, pp. 4022-4042, 2020.

[3] J. Liu, "Study on the collaborative governance mechanism of ecological environment in Changsha-Zhuzhou-Xiangtan
Region under the green development perspective," Meteorological and Environmental Research, vol. 9, no. 5, pp. 51-55, 2018.

[4] J. W. Park, "The role of environmental law in assessing and managing risk of chemical products and biocides," Environmental Law and Policy, vol. 20, no. null, pp. 55-85, 2018.

[5] P. Hassan, "Role of the south in the development of international environmental law," Chinese Journal of Environmental Law, vol. 1, no. 2, pp. 133-157, 2017.

[6] Y. Zhao, "International governance and the rule of law in China under the belt and road initiative," The Role of Environmental Impact Assessment in the Governance of the NuSalween River, Cambridge University Press, Cambridge, UK, pp. 218-242, 2018.

[7] J. R. M. Leite and M. D. Venâncio, "Environmental protection in Brazil's High Court: Safeguarding the environment through a rule of law for nature," Seqüência: Estudos Jurídicos e Políticos, vol. 38, no. 77, pp. 29-50, 2017.

[8] W. Cao, "On the methodological consciousness in the codification of environmental law\%," Journal of Renmin University of China, vol. 033, no. 002, pp. 39-51, 2019.

[9] P. Mccoy, "How environmental protection reaches the public eyes and ears: The environmental journalism of environmental law and politics," Diseases of Aquatic Organisms, vol. 48, no. 2, pp. 133-142, 2017.

[10] X. B. Li, "Study of Funan willow art cultural characteristics under the background of cultural heritage protection," Journal of Huaihua University, vol. 36, no. 4, pp. 13-15, 2017.

[11] C. Zhao, S. M. Zhong, Q. S. Zhong, and K. B. Shi, "Synchronization of Markovian complex networks with input mode delay and Markovian directed communication via distributed dynamic event-triggered control," Nonlinear analysis. Hybrid systems, vol. 36, p. 100883, 2020.

[12] M. P. Gao, L. Nie, and X. Z. Qi, "Study on the influence of the environmental protection tax law of the People's Republic of China on the coatings industry," China Coatings, vol. 33, no. 1, pp. 12-21, 2018.

[13] H. Shaikh, X. Deng, and Z. Li, "Projections of future land use in Bangladesh under the background of baseline, ecological protection and economic development," Sustainability, vol. 9, no. 4, 505 pages, 2017.

[14] A. P. Zhou, "Research on circular using of resource in the view of ecological environmental protection," Journal of Yellow River University of science and technology, vol. 19, no. 4, pp. 71-75, 2017.

[15] Y. Lin, H. Song, and F. Ke, "Optimal caching scheme in D2D networks with multiple robot helpers," Computer Communications, vol. 181, pp. 132-142, 2022.

[16] Y. Jiang and X. Li, "Broadband cancellation method in an adaptive co-site interference cancellation system," International Journal of Electronics, 2021.

[17] N. Craik, C. Jefferies, S. L. Seck, and T. Stephens, "Global Environmental Change and Innovation in International Law," Climate Change and Protection of the Marine Environment, Cambridge University Press, Cambridge, UK, 2018.

[18] J. Dong, Y. Cong, and G. Sun, "Where and how to transfer: Knowledge aggregation-induced transferability perception for unsupervised domain adaptation," IEEE Transactions on Pattern Analysis and Machine Intelligence, p. 1, 2021.

[19] X. Liang, T. Lu, and G. Yishake, "How to promote residents' use of green space: An empirically grounded agent-based modeling approach," Urban Forestry \& Urban Greening, vol. 69, Article ID 127435, 2022. 